\title{
AUTOMATIC FIRE DETECTION SYSTEM BASED ON CONTOUR ANALYSIS VIDEO IMAGES
}

\author{
Irina Egoshina ${ }^{1}$, Dmitry Titov ${ }^{2, a}$, Anton Stuchkov ${ }^{3}$ \\ ${ }^{1}$ Volga State University of Technology,424000, the Republic of Mari El, Yoshkar-Ola, Russia \\ ${ }^{2}$ Southwest State University, 405000, Kursk, Russia \\ ${ }^{3}$ Tomsk State University, 634050, Tomsk, Russia
}

\begin{abstract}
A general structure of an automatic fire detection system and the results of a study video analysis algorithms for detecting based on contour analysis. Given contour extraction processes objects in the image and make decisions about the discovery of fire or the absence thereof. Experimental researches on the video images of the forest area done with the use of the developed structure of the automatic fire detection system.
\end{abstract}

\section{Introduction}

The efficiency of the fire problem depends from the early detection of the fire place. Usually space monitoring and aviation are using for the decision of this actual problem. Every method has its benefits and weaknesses, but only the automatization of the work of the territory monitoring system, which should determine and automatically recognize the fire place in the early beginning, and then information of the necessary services can solve the problem in the area of fire safety. That's why the development of the automatic fire detection system based on modern intellectual technologies is a very important task nowadays.

The development of modern technologies of the technical vision allows determining the fire on digital images in the automotive way. Such systems with the use of different sensors get the data about the environmental condition and analyze the fire probability by the determination of such dangerous visual objects as smoke or fire without the operator work and inform him just after getting the final decision about real danger [1,2].

\section{Structure of the automatic system and methods of determination of dangerous fire factors}

Developed system is made for determination of the fire and its' fireplace in the real-time mode. The existing info communication framework of the data transfer can be used for the system functioning.

Enlarged structure of the automation system consists of these functional blocks (Figure 1):

\footnotetext{
${ }^{\text {a }}$ Corresponding author: amazing2004@inbox.ru
} 
- system of sensors;

- software block;

- fire alarm system;

- PC operator.

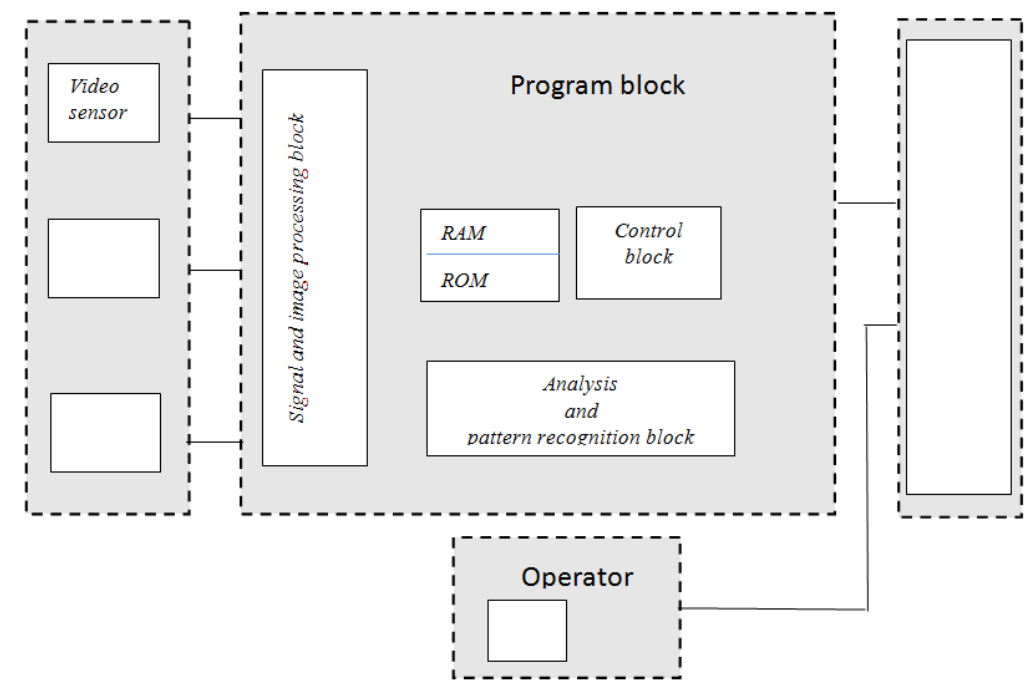

Figure 1. Enlarged structure of the automatic fire detection system.

The system of sensors, which includes video sensors, infrared sensors, ultraviolet sensors, controls such parameters as the increasing of the air temperature, appearance of the open fire, detection of certain changes in the video image. Because of the simultaneous use of the ultraviolet and infrared channels the amount of fireplaces, their location and fire intensity can be detected. The use of three types of determination channels and of the special algorithm of the signal and image processing will decrease the possibility of false operations and more effective control the situation in the zone of the system work [3].

Software block has such functions as the preliminary signal and image processing from the sensors; archiving of the video image; storage of the database for determination of the fire objects; analysis and fire detection. Control block allows to get the final decision of the start of the fire on the intellectual level, i.e. the control signal will go to the fire alarm system after analyzing of several object parameters.

The task of the operator in this process is to react timely on the system signals.

Basic fire factors which can be registered by the video sensor and preventing factors are described down [4,5]:

- $\quad$ Open flame differs highly from the backlight by the intensity and frequency of the electromagnetic radiation. Sometimes the flame can be determined by the video sensor, based on the special algorithm of the signal processing. The advantage is the possibility to control of big open grounds. The additional advantage is the possibility to fix the exact place of the fire in the automotive mode. Preventing factors are noises from the sun, changes of the time of the day, rainfalls, different movements of people in the frame, but there is no problem to fix this factors. The technical difficulty can cause during the creation of the special video sensor with a wide dynamic range. 
- A column of smoke on the open ground can be easily determined by the strong change of the image contrast on the big size of the image. In this case same problems appear as in the previous one.

- Indoor smoke: firstly smoke appears in the upper part of the space in the very beginning of the fire. The main preventing factor in this case can be insufficient contrast in the space in the dart time of the day, but this problem can be solved by the use of "reference signals" or the use of the infrared sensors.

Well known methods and developed on their basis algorithms of the video signal processing, which can be used in determination of dangerous factors of the fire, can be divided in 3 groups [6-8].

The first group includes methods, using references images, gotten before the dangerous situation in normal conditions in different types of illumination (day, evening, rain, etc.). The second group includes methods, using the exact database of the typical video image blocks of such different dangerous situations as the form and the type of the flame, intensity of the smoke and different preventing factors, such as the solar flare, lights of moving cars, and different types of flares. The third group includes methods, which analyze static and dynamic components of image elements by brightness and color components, and also with the use of special sensors of the infrared range.

In this work the method and algorithm of the fire factors determination based on the contour analysis of video images are described. Contour approach allows not to analyze inside points of the image and because of that to decrease the size of processed data by the movement from the function with two variables to the function of the one variable. The consequence of this is the possibility of work of the system in the real-time mode.

\section{Algorithm of the image analysis for the fire detection based on the contour analysis}

Described automatic system includes the records of video images of the controlled surface and contour standards of the image objects. The result of work of the signal and image processing block is the basic analysis of the image characteristics (brightness, contrast and others, which depend from the peculiarities of the shooting and parameters of the equipment) and their preliminary processing [9-12].

Analysis and pattern recognition block by the gotten video images of objects can react in a such way:

- Detection of the object contour on the image.

- Formation of the scalar product of the recognizing contour with the standard contour.

- Comparison of the real part of the scalar product with the threshold value.

- Getting the final decision about the fire determination.

Generally, the algorithm of detection is as follows. We have standard contour $\mathrm{G}$ of the image object of the controlled surface before dangerous situation and determined contour $\mathbf{N}$ of the object in the current image. Standard can be formed depends from the weather situation, illumination, etc.

In this case the algorithm of detection will look:

$$
\operatorname{Re}(\mathbf{N}, G) \geq \sigma^{2} \ln \Lambda_{0}+0,5\|G\|^{2}=U_{0},
$$

where $\Lambda_{0}$ is the threshold value, $U_{0}$ is the threshold, which depends from the energy of the standards and noise contours and risks. 
Thus, for getting the final decision about the fire detection in the exact place the object contour $\mathbf{N}$ should be determined and scalar product of the determined contour $\mathbf{N}$ with the standard contour $\mathbf{G}$ should be done. Then the real part of the scalar product is comparing with the threshold value, depends from the energy difference, noise dispersion, risks [13].

Researches in the fire detection were made on the example of video images of the forest area (Figures 2-4). The object contour was determined on the image (the upper level of the forest area) and then was compared with the standard contour of the exact area (before the dangerous situation).

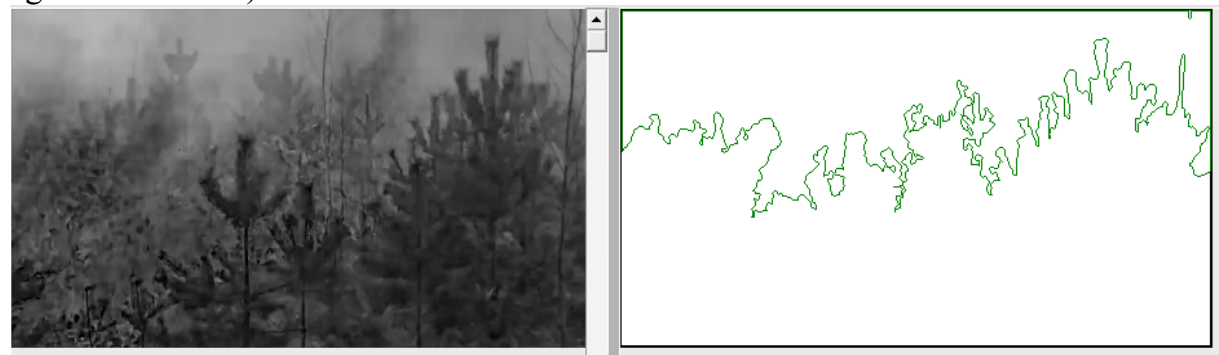

Figure 2. Video image and the formed standard contour of the object $G$ (upper level of the forest area).
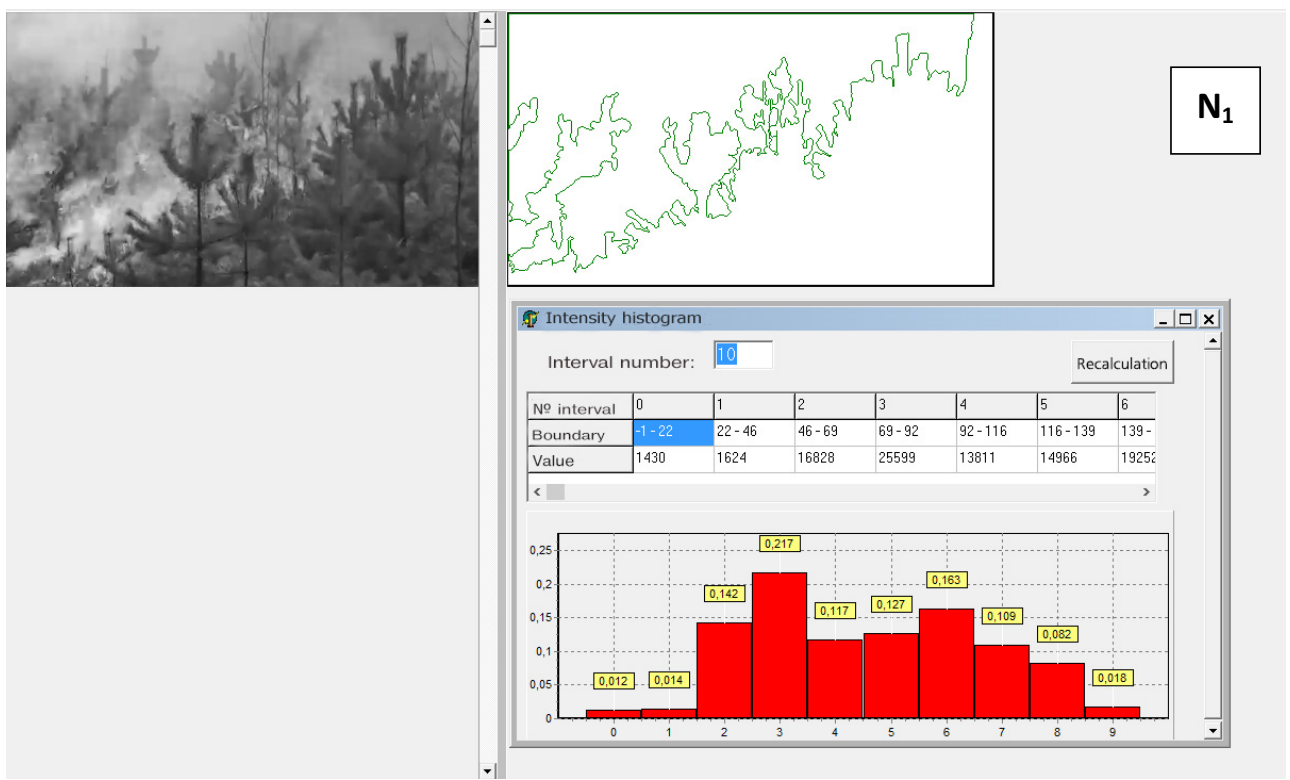

Figure 3. Video image of the forest area before the appearance of the dangerous situation on the exact are and it's processing (contour $\mathrm{N}_{1}$ is determined). 

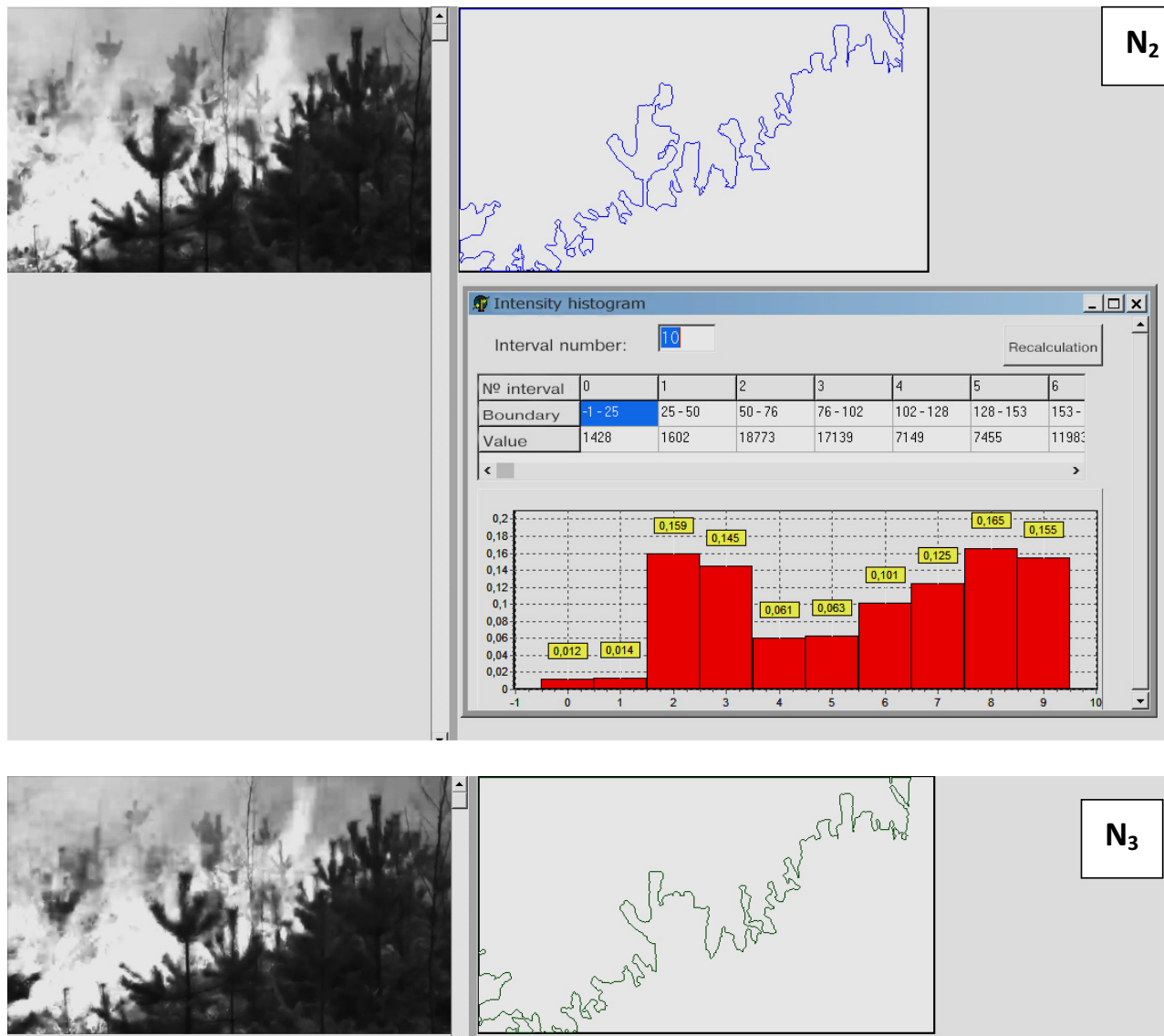

$\mathbf{N}_{3}$
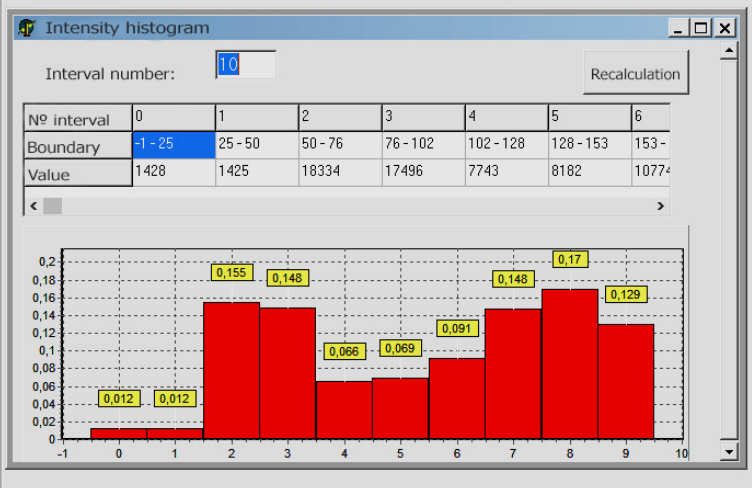

Figure 4. Video images of the forest area during the fire and their processing with interval 5 seconds (contours $\mathrm{N}_{2}, \mathrm{~N}_{3}$ are determined).

\section{Conclusion}

Experimental researches, which were made on the video image of the forest area, shown that during the fire appearance the object contour is changing during the all dangerous situation. The results of the developed algorithm show that in the image with determined contour $\mathbf{N}_{\mathbf{1}}$ the final decision about the fire absence is done, in images with determined contours $\mathbf{N}_{2}, \mathbf{N}_{3}$ the final decision about the fire appearance is done. 
Thus, contour analysis can be shown as the mathematical model, which provides the way for decision of the problem of the image processing and the private problem of the fire appearance and dangerous situations on video images.

\section{Acknowledgements}

The paper was written as part of the research project No. 8.2.31.2015, carried out with the support of the Program "Research Foundation of Tomsk State University named after D.I. Mendeleev" in 2015 - 2016, grant RFBR No. 16-29-04388/16.

\section{References}

[1] A. Kuznetsov, V. Musalimov, A. Saenko, K. Trambitskiy, Journal of Instrumental Engineering 55, 51 (2012)

[2] M.V. Rukin, Safety systems (4), 61 (2009)

[3] C. Koulas, Proceedings of SPIE - The International Society for Optical Engineering 7298, 72983Q (2009) doi: 10.1117/12.818987

[4] I Y. Furman, A. Krevetskiy, A. Peredreev, A. Rozhentsov, R. Hafizov, I. Egoshina, A. Leushin, Introduction in the contour analysis and it's applications for the image and signal processing ( Fizmalit, Moscow, 2003)

[5] H. Kennedy, Opt. Eng. V. 30 , 1771 (1999)

[6] N. Koshchavtsev, Proceedings of SPIE - The International Society for Optical Engineering 4369, 81 (2001) doi: 10.1117/12.445278

[7] N. Topolskiy, A. Chlenov, T. Butcinskaya, M. Zemljanukhin, International workshop "Built Heritage: Fire Loss to Historic Buildings" (2004)

[8] E. Wixon, H. Rocky, Method and apparatus for determing ambient conditions from an image sequence, such as fog, haze or shadows, US patent no. 6037976 (2010)

[9] T. Yamagishi, M. Kishimoto, Fire detection system utilizing relationship of correspondence with regard to image overlap, US patent no. 5926280 (1999)

[10] R. Borsh, Imaging fire detector WO patent no. 01/67415 (2001)

[11] D.V. Shashev, S.V. Shidlovskiy, V.I. Syriamkin, A.V. Yurchenko, IOP Conference Series: Materials Science and Engineering 81, 012101 (2014) doi: 10.1088/1757-899X/81/1/012101

[12] D.V. Shashev, S.V. Shidlovskiy, Optoelectronics, Instrumentation and Data Processing 51, 227 (2015) doi: 10.3103/S8756699015030036

[13] M. Eisman, Proceedings of SPIE - The International Society for Optical Engineering 5783, 71 (2005) doi: 10.1117/12.606718 